\title{
Interseccionalidad, jóvenes "sin-sistema” y resistencia. Una mirada diferente del fracaso/abandono escolar
}

\author{
Rosa Vázquez-Recio' (D) \\ Mónica López-Gil' iD
}

\section{RESUMEN}

El fracaso y abandono escolar constituyen unos de los temas que suscita mayor preocupación, tanto a nivel nacional como internacional. Se trata de un fenómeno sobre el que existe una amplia tradición de estudios e investigaciones que nos ofrece el mapa de factores que intervienen en la concomitancia del fracaso y abandono. Sin embargo, entendemos que, pese al valor de estos aportes, sigue existiendo un vacío de experiencias contadas por los sujetos que se encuentran en esa situación. En este plano se sitúa este trabajo que emerge de las reflexiones derivadas del proyecto de investigación "Riesgo de fracaso y abandono escolar en Secundaria Obligatoria. Los contextos educativos, familiares y socioculturales. Un estudio cualitativo". Tomamos como herramienta de análisis el concepto de interseccionalidad y matriz de dominación de Hill Collins que nos permite hablar de los jóvenes "sin-sistema" y de su resistencia al sistema escolar y a las instituciones educativas.

\section{PALABRAS CLAVE}

fracaso escolar; abandono escolar; educación secundaria; interseccionalidad; matriz de dominación; reconocimiento.

'Universidad de Cádiz, Cádiz, España. 


\section{INTERSECTIONALITY, YOUNG PEOPLE “WITHOUT- A-SYSTEM" AND RESISTANCE. A DIFFERENT LOOK AT SCHOOL FAILURE/DROPPING OUT}

\section{ABSTRACT}

Failure and dropping out of school are some of the issues that cause the greatest concern, both nationally and internationally. It is a phenomenon on which there is a long tradition of studies and research that offers us the map of factors that intervene in the concomitance of failure and abandonment. However, we understand that, despite the value of these contributions, there is still a vacuum of experiences told by the subjects who are in that situation. This work is part of this plan that emerges from the reflections derived from the research project "Risk of failure and school dropout in Secondary Education. The educational, family and sociocultural contexts. A qualitative study". As an analytical tool we use the concept of intersectionality and dominance matrix of Hill Collins that allows us to talk about young people "without-a-system" and their resistance to the school system and educational institutions.

\section{KEYWORDS}

school failure; dropping out; secondary education; intersectionality; matrix of domination; recognition.

\section{INTERSECCIONALIDADE, JOVENS "SEM-SISTEMA" E RESISTÊNCIA. UM OLHAR DIFERENTE DO FRACASSO/ABANDONO ESCOLAR}

\section{RESUMO}

O fracasso e o abandono da vida escolar são algumas das questões que causam a maior preocupação, tanto nacional como internacionalmente. Trata-se de um fenômeno em que há uma longa tradição de estudos e pesquisas que nos oferecem o mapa de fatores que intervêm na concomitância do fracasso e do abandono. No entanto, entendemos que, apesar do valor dessas contribuições, ainda há um vazio de experiências contadas pelos sujeitos que estão nessa situação. Este trabalho surge neste âmbito, realizado a partir das reflexões derivadas do projeto de pesquisa "Risco de fracasso e abandono escolar na Educação Secundária Obrigatória. Os contextos educacional, familiar e sociocultural. Um estudo qualitativo". Como ferramenta analítica, utilizamos os conceitos de interseccionalidade e matriz de dominância de Hill Collins, que nos permite falar sobre jovens "sem-sistema" e sua resistência ao sistema escolar e às instituições educativas.

\section{PALAVRAS-CHAVE}

fracasso escolar; abandono escolar; ensino secundário; interseccionalidade; matriz de dominação; reconhecimento. 
El mundo estaba perdido en muchos pedazos idos.

Los demás fueran afueras de tantos mundos caídos.

Ya nunca, ya nadie vino. Ya nadie pudo poner Iguales pedazos juntos, Tantos mundos en su sitio

"Infancia: elegia" (Valente, 2016, p. 241)

\section{MIREMOS A TRAVÉS DEL ESPEJO: ¡ABRE LOS OJOS!}

Este trabajo se presenta como un desafío que asume una mirada política sobre lo que se viene conceptualizando como fracaso y abandono escolar, en la medida en que dicha mirada coloca las experiencias de los jóvenes afectados por el fracaso escolar, que en muchos casos terminan abandonando el sistema educativo, en el centro del análisis de tales fenómenos. No se trata de dotarles de privilegios frente a toda la serie de factores que son identificados en la concurrencia de los mismos, sino de hacer un esfuerzo por intentar comprenderlos desde el reconocimiento de quienes los experimentan en primer término, porque, digámoslo claramente, cuando se pronuncia la palabra "fracaso escolar" la imagen que evoca o la idea que emerge de golpe es la que corresponde al "alumnado", y no a aspectos que tengan que ver con metodologías no inclusivas, con un sistema educativo incapaz de dar soluciones, con una política educativa basada en estándares y en rendición de cuentas o con un sistema social mercantilizado promotor de desigualdades. En palabras de Gimeno Sacristán (2003, p. 17),

preocupa el fracaso escolar, pero no tanto "los fracasados" [...] generalmente, cualquier problema o tema de discusión en educación tiene a sus receptores como un referente implícito; en muchas menos ocasiones el sujeto escolarizado aparece en el centro de las controversias. Quienes más presencia tienen en el sistema escolar pueden quedar de esa forma diluidos en el discurso, ausentes en nuestras conciencias y relegados a nuestras preocupaciones [...]. Hablar de neoliberalismo, de comprensividad o de calidad del sistema educativo es hacerlo de orientaciones, preocupaciones o propuestas que afecten a los estudiantes, pero es frecuente no encontrarlos en estas discusiones.

Y en este tomar en primer plano las experiencias de los "fracasados", hemos de recordar lo que acertadamente argumentaba Perrenoud (1996): el fracaso no existe por natura, sino que es un artificio, una construcción que deviene de la posición que se ocupa dentro de la jerarquía de excelencia que se fabrica con una carga ideológica y con implicaciones políticas que marcarán las decisiones que se tomen al respecto. Hablamos del fracaso desde fuera de este para otorgarle un estatus desde el que nos 
sea posible encontrar explicaciones y justificaciones, pero no desde dentro, es decir, desde los propios sujetos que experimentan ese proceso continuado y progresivo de desenganche que desemboca en fracaso y/o abandono escolar. El modo en el que pensamos e intentamos explicar y comprender este fenómeno marca la diferencia, y la señalamos asumiendo una mirada que se nutre del concepto de interseccionalidad trabajado por Hill Collins (1990, 2009).

Cuando atendemos a las tasas de fracaso y abandono escolar, no podemos pensar que se vayan a reducir o anular con repeticiones de curso, horas de refuerzo o apoyo, o con un profesorado más comprometido con la inclusión. Mientras que se mantenga intacto un sistema educativo orquestado por políticas educativas neoliberales y conservadoras, que se gestionan y se articulan conforme los requerimientos que establece la ideología de las élites dominantes que gobiernan el mundo en todos sus ejes (economía, cultura, educación, etc.), será difícil dar solución al fracaso escolar, porque, además, interesa que exista para poder justificar la necesidad de acometer reformas educativas que sigan reforzando los ideales de emprendimiento, esfuerzo y excelencia a altas cotas; es, utilizando el calificativo de Enrique Martínez Reguera (2007, p. 17 y ss.), muy rentable.

Los estándares que se establecen para el logro de buenos resultados o de un rendimiento académico considerado de calidad solo sirven para mantener y legitimar a los grupos y a los sujetos que ya disfrutan del "privilegio" de encajar en el sistema escolar; la meritocracia impuesta por la ideología hegemónica repercute en la equidad imposibilitándola, pero abonando el terreno para el fracaso y el abandono (Tarabini, 2015). Mientras que los sistemas, tal cual están configurados, sigan beneficiando a los "privilegiados" (es decir, aquellos que no pertenecen a la categoría de excluidos), cualquier tentativa de cambio resultará irrelevante para los que sufren una violencia estructural, que son los que sí están incluidos en dicha categoría. La desatención y la ausencia de actuaciones convierten a los jóvenes en situación de riesgo de fracaso y/o abandono en sujetos "sin-sistema". ${ }^{1}$ Sus voces son silenciadas, pero este silenciamiento no los convierte en sujetos pasivos, sino en actores que desarrollan estrategias que se muestran como gestos de resistencia (Willis, 1988), entre ellas, el silencio o no hacer nada. Pese a que parece que se está promoviendo una educación inclusiva, equitativa y participativa desde estancias diversas, entre ellas, la administración educativa, en realidad lo que se viene produciendo es una enunciación que encubre prácticas discursivas y regulaciones normativas despolitizadas que perpetúan la exclusión educativa, también es de carácter político (Young, 2000), en un escenario aparentemente inclusivo y democrático. Y resulta de tal modo en cuanto que en ese escenario confluyen discursos $\mathrm{y}$ acciones complejas que generan desigualdades y margina, de manera diferente, al alumnado que no "encaja" en el sistema, impidiéndole participar y a disfrutar de las oportunidades que le correspondería como sujeto de un Estado de bienestar.

Esta mirada política que hemos asumido en este trabajo viene a ser el resultado de las reflexiones realizadas a partir de la investigación que venimos desarrollando en el marco del proyecto de investigación "Riesgo de fracaso y abandono escolar en

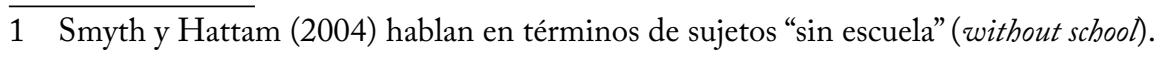


Secundaria Obligatoria. Los contextos educativos, familiares y socioculturales. Un estudio cualitativo" (Junta de Andalucía, SEJ-2664); ${ }^{2}$ reflexiones que se concretan en un desarrollo teórico argumentativo y no en el aporte específico de resultados. Esta mirada parte del reconocimiento de la complejidad que caracteriza a los fenómenos del fracaso y del abandono escolar, hecho que implica la retirada de la idea monolítica del "problema es del alumnado" y la necesidad de incorporar marcos interpretativos que asuman el carácter interseccional que define e interviene en la construcción del fracaso y del abandono escolar. A estas cuestiones nos dedicaremos en las siguientes líneas.

\section{LA LÓGICA METODOLÓGICA ASUMIDA COMO COMPROMISO}

La metodología adoptada ha sido la investigación cualitativa realizando entrevistas etnográficas semiestructuradas (un total de 91, con una presencia del $33 \%$ de mujeres y del $67 \%$, de hombres), estudios de caso biográficos (9) y grupos focales (3). El trabajo de campo se ha realizado en las provincias andaluzas de Cádiz, Málaga y Almería, España. Los sujetos informantes pertenecen al intervalo de edades comprendidas entre 12 y 26 años: el 46,3\% de las personas entrevistadas tienen entre 12 y 16 años (grupo que tiene que permanecer en el sistema educativo, y cursa educación secundaria obligatoria/ESO), y el 53,7\% con edades comprendidas entre 17 y 26 años (ya sin la obligatoriedad de permanencia, realizando formación profesional básica, formación profesional de grado medio y grado superior, programas de cualificación profesional inicial/PCPI, educación secundaria para personas adultas/ ESA). Con relación a los grupos, nos ha interesado tanto el alumnado en riesgo y que ha abandonado temprano el sistema, como aquel que ha retomado los estudios cursando la ESA y otros como los universitarios. Para el tratamiento de los datos se ha utilizado el software Nvivo11 que han permitido establecer tres potentes categorías: el contexto escolar (especialmente destacable el profesorado), el contexto familiar, el sistema educativo y los rasgos de identidad. La gestión de la calidad de la investigación se ha garantizado mediante la triangulación entre los diferentes procedimientos y agentes de investigación. Igualmente, los principios éticos que han guiado este proceso indagatorio han sido, fundamentalmente, la negociación, la confidencialidad, la participación y el compromiso con el conocimiento (Vázquez-Recio, 2014).

\section{“iNO ESTOY LOCO! MI REALIDAD ES SIMPLEMENTE DIFERENTE A LA TUYA!”3}

Fracaso y abandono escolar forman parte de nuestro imaginario social con todas las atribuciones que les corresponden, las cuales proceden de focos diferentes,

2 El proyecto de investigación está siendo desarrollado en las provincias andaluzas de Cádiz, Málaga y Almería en el marco de la convocatoria de excelencia de la Consejería de Innovación, Ciencia y Empresa de la Junta de Andalucía, y bajo la dirección actual de Rosa Vázquez-Recio de la Universidad de Cádiz.

3 Carroll, L. Alicia en el País de las Maravillas. A través del espejo. Madrid: Akal, 2003. 
pero que, finalmente, actúan de manera complementaria incrementando su poder determinante sobre los sujetos sobre los que recaen tales etiquetas. Por una parte, los trabajos que ofrecen toda una serie de factores que hacen converger para establecer posibles correlaciones con las que poder fijar un modelo explicativo del hecho en sí llamado fracaso y abandono escolar, que, aun siendo de valor tales aportes, terminan ofreciendo una visión de dichos fenómenos descarnada y descontextualizada de las realidades que dan cuenta de los mismos (en esto ha jugado un papel importante la investigación cuantitativa). Por otra, ese imaginario no se puede sostener sin un lenguaje que ayude a construirlo y a mantenerlo; un lenguaje que no está desprovisto de los ingredientes precisos para representar un fenómeno que termina convertido en vox populi desde una mirada sesgada y negativa. Cuando de alguien se dice que es un fracasado o que ha fracasado, las expectativas que conlleva tal enunciación no son favorables; se piensa en clave de "qué se puede esperar de ese alguien", y lo esperado suele ser más bien poco que mucho. Nuestro pensamiento fracasa porque nuestro lenguaje contribuye a configurar las situaciones y la vida de ese alguien bajo una serie de premisas falsamente infundadas en muchas ocasiones, $y$ lo son porque nos manejamos con supuestos implícitos cargados de valores, prejuicios, estereotipos y representaciones que se tornan en certezas o verdades. Dichos supuestos nos llegan por canales hegemónicos que están bajo el control de las estructuras de dominación; certezas o verdades que no son cuestionadas.

"Una palabra es suficiente para hacer o deshacer la fortuna de un hombre", decía Sófocles, y la palabra "fracaso" - a la que le añadimos "escolar" para perfilarla y delimitar su campo de acción y efecto - es una de esas palabras que quebranta la comprensión de un "sí mismo" en continuo proceso de construcción e interpretación; palabra que rompe el porvenir, las ilusiones, los deseos de ese alguien que, por ser alguien, ya pasa al anonimato. A este estatus de alguien es al que pasan esas chicas y esos chicos adolescentes que son considerados fracasados porque han fracasado, y lo son porque no han alcanzado los resultados previstos y esperados por el sistema educativo. Es un estado de indeterminación en el que no caben preguntas como: quién es ese alguien, cómo se llama, qué le gusta, a qué aspira, cuáles son sus costumbres, qué siente, qué necesita, cómo vive y en qué condiciones lo hace, etc.; un estado de indeterminación que le exige y obliga a perder sus atributos y a asumir aquellos otros que se entienden que son los necesarios para responder a las exigencias del sistema escolar y a la sociedad a la que pertenece (pero con los que no se identifica porque no responden a sus necesidades y prioridades); un estado en el que se amputa el intento de la escucha y del reconocimiento del otro como parte de un "nosotros"; un estado que le prohíbe su derecho a ser quien es, porque como es (su "yo" " "sus circunstancias", su cultura) no se ajusta al molde tallado en el que ha de encajar; un estado sobre el que recae la exclusión moral (Fallis y Opotow, 2003, p. 112) al no ser considerados dignos de equidad o del derecho del disfrute de los bienes de un Estado de bienestar.

Pero este estado que le arrastra al silencio, a la invisibilización y al estigma, también, y al mismo tiempo, le potencia y/o refuerza su capacidad de resistencia, porque, como señala Willis (1988), existe. El alumnado se resiste recurriendo a estrategias que no son aceptadas ni reconocidas como éticamente correctas (peleas, 
agresiones, réplicas, insultos, no realizar las tareas, llegar tarde al centro, molestar a los compañeros en clase o en otros espacios, etc.); estrategias que podemos considerar subversivas en algunos casos. Una resistencia que se puede considerar legítima ante esa cultura que legitima la institución escolar y el sistema educativo que no conecta con sus necesidades y dificultades de su vida cotidiana; una institución y un sistema que absorben cualquier tentativa, experiencia, visión que difiere de la cultura oficial al mostrarse distinta y alternativa si ha lugar. Cabe preguntarnos: $¿$ No tiene derecho a enfadarse, a revelarse cuando percibe que algunos profesores no tienen paciencia, cuando el diálogo se torna en chillidos y gritos, cuando hay desmotivación, rechazo, insultos, aburrimiento, indiferencia, cuando le "tratan como a una basura", ${ }^{4}$ cuando recibe mensajes como "nunca vas a aprobar", 5 "no te cachondees [referido a la maestra] porque te estoy preguntando, no es nada de otra cosa", ${ }^{6}$ o cuando "por nada me ponía un parte, decía 'para abajo' por nada que hacía para abajo, hiciera lo que hiciera para abajo?" Martínez Reguera (2007, p. 24), tras cuarenta años de trabajo con jóvenes inmersos en problemas sociales y educativos, comprobó la férrea resistencia de ellos:

tal vez sea lo que más tardé en comprender y para lo que estaba peor preparado: para entender que nuestras mejores intenciones, dentro del marco en que nos movemos, puedan ser peligrosas para ellos. Tardé mucho en entender lo legítimo y necesario que pudiera ser el que los chavales se defiendan de nuestras buenas intenciones.

La resistencia no deja de ser una respuesta ideológica ante el aparato ideológico escolar que impone una lógica homogenizadora y estandarizada que evidencia, a su vez, que dentro de las estructuras de dominación hegemónicas, la pasividad no es el efecto exclusivo que logra, sino también la acción rebelde, la contestación, la desobediencia, la conducta de oposición, el desafío, etc., so pena de que esto conlleve consecuencias negativas que, en algunos casos y como apunta Calderón Almendros (2014), implique la propia destrucción. Esa resistencia no es estática y uniforme porque es contextual (Haenfler, 2004, p. 409), dado que el riesgo (at risk) esa situación a la que se encuentran expuestos algunos estudiantes caracterizada por factores de riesgo y/o condiciones particulares que hace que estos tengan más probabilidad de tener consecuencias adversas o desfavorables (Finn y Rock, 1997, p. 221-222) - es una construcción en la que intervienen las estructuras escolares y sociales injustas y discriminatorias (Vasudevan y Campano, 2009, p. 315). Por tanto, el riesgo de "caer" en el fracaso y el abandono no es una condición inmanente de

4 Testimonio de una adolescente de 17 años, repetidora de $4^{\circ}$ de educación primaria y $1^{\circ}$ de ESO.

5 Testimonio de un joven de 18 años, repetidora de $6^{\circ}$ de educación primaria y $2^{\circ}$ de ESO (actualmente cursando $1^{\circ}$ de ESA).

6 Testimonio de un adolescente de 15 años, repetidor de $2^{\circ}$ y $4^{\circ}$ de educación primaria (cursa $2^{\circ} \mathrm{ESO}$ ).

7 Testimonio de un adolescente de 15 años, repetidor de $2^{\circ}$ de educación primaria y $2^{\circ}$ de ESO (cursa segundo año de $2^{\circ}$ ESO). 
los estudiantes que terminan cayendo, y consecuentemente, al no serlo, no puede asumirse como una condición que no pueda ser cambiada (Fallis y Opotow, 2003; Lee, 2009) con pedagogías inclusivas y prácticas innovadoras.

Pensar en clave de resistencia nos puede ayudar a conocer ante qué situaciones (tareas en clase frente a las realizadas fuera, por ejemplo), contextos (con amigos o no, estar con los compañeros de siempre o pérdida de estos), códigos (de relación, comunicación, expresión, argumentación, etc.) y profesorado (según disciplinas curriculares), ${ }^{8}$ el alumnado adopta unas estrategias u otras de resistencia para hacer frente a la indiferencia ("yo pregunto una duda, y a mí no me hacen tanto caso como $[\ldots]$ a otra persona que estudia bastante"), ${ }^{9}$ a la valoración negativa ("yo no sirvo para nada, que no sirvo para nada, que yo no sirvo para nada, me decían"), ${ }^{10} \mathrm{a}$ códigos elaborados que quedan fueran de su alcance ("y no hacer nada en la clase ni nada $[\ldots]$ porque hay algunos maestros que explicaban y como yo no sabía lo que decían, pues me quedaba ahí dormida, daba cabezadillas"), ${ }^{11} \mathrm{o}$ a otras tantas circunstancias que llegan a tener repercusión en la población adolescente que no “encaja”. Como señala Hill Collins (2009), es necesario pensar de manera amplia la resistencia para descubrir en qué dominio pueden tener mayor efecto el resistir a los sistemas que ejercen poder y opresión sobre los sujetos y cómo estos lo afrontan.

\section{ENTONCES, ¿DÓNDE COLOCAR EL ARTIFICIO DEL FRACASO? POSICIONES SUBALTERNAS DE LOS JÓVENES “SIN-SISTEMA"}

Visto desde esta panorámica, cabe preguntarse hasta qué punto el fracaso les pertenece a esos chicos y esas chicas aunque se muestren como infractores del orden y de las reglas establecidas, desviándose así de lo institucionalizado. Con demasiada facilidad se pierde una visión holística e interseccional del fenómeno del fracaso, cuyo efecto es la focalización del mismo en el sujeto que encarna el mismo y que lo proyecta en sus contextos sociales, educativos y familiares. Parece que sigue teniendo vigencia el llamado "efecto pararrayos" con el que se explicaba la necesidad de los "idiotas" y de los "tontos" para liberar al resto no incluido en estas categorías sociales. De manera sutil y pasando inadvertida con elegancia, esta focalización significa que se ha producido el desplazamiento del "fracaso" desde el propio sistema educativo, que actúa bajo pedagogías invisibles y estructuras organizativas tecnoburocratizadas, al sujeto (estudiante). Con ello da la sensación de que el sistema no toma parte en la gestación del fracaso y del abandono escolar, ni tampoco existe el gesto firme y claro por comprender lo que está ocurriendo y afectando a un porcentaje de adolescentes (que empiezan a sufrir los síntomas en la educación

8 En las disciplinas en las que el alumnado muestra más resistencia son en lengua, matemáticas, biología, física, química e historia.

9 Testimonio de un adolescente de 15 años, repetidor de $4^{\circ}$ de educación primaria y $1^{\circ}$ de ESO.

10 Testimonio de un adolescente de 15 años, repetidor de $1^{\circ}$ y $2^{\circ}$ de ESO.

11 Testimonio de una adolescente de 16 años, repetidora de $2^{\circ}$ de educación primaria, $1^{\circ}$ y $2^{\circ}$ de ESO. 
primaria y que son los desencadenantes de situaciones de riesgo - at risk - en la educación secundaria). Los relatos de los jóvenes - como los obtenidos en nuestra investigación - evidencian que la educación comprensiva e inclusiva no se está haciendo realidad en las instituciones educativas; los "otros", esos alguien que están incluidos en la categoría de "fracaso escolar" siguen resultando ser el colectivo excluido y buenamente desatendido.

Este excluirse el sistema educativo de estas situaciones - y todo lo que este implica en sí como concreción de las exigencias del orden social, económico y político- equivale a decir que la política educativa, las regulaciones normativas, el profesorado, las medidas que se establecen, la estructura organizativa de los centros, etc., no intervienen en la causación de tales problemáticas y, por tanto, no se va a poner en cuestión que lo que se está planteando sea algo equívoco o que no sea lo más adecuado. Como tampoco se ponen en valor los efectos que está teniendo la sociedad de mercado, competitiva y de consumo en ese sector de la población escolarizada en el que está provocando fracturas tanto personales, familiares y sociales. Estos efectos son perversos ya que dinamitan el tejido social que garantiza el bienestar común del que ha de disfrutar la ciudadanía sin discriminaciones y exclusiones. El resultado de ello es una masa de sujetos agregados que se ven abocados a la búsqueda, al precio que sea, del éxito para poder responder así a la ideología legitimadora de la política hegemónica de la econometría, la rentabilidad y los beneficios.

Esa masa, que los condicionantes de las políticas neoliberales y conservadoras hacen que cada vez esté más desprovista de compromisos y obligaciones sociales, políticas y éticas, es una masa vacía llena de un individualismo competitivo, clasista y segregador que impone en su seno una categorización de sujetos que demarca los "sujetos fuertes" de los que son "sujetos débiles", los "sujetos ejemplares" de los "sujetos indignos", los "sujetos responsables" de los "sujetos irresponsables", los "sujetos sensatos" de los "sujetos insensatos", los "sujetos buenos" de los "sujetos malos", y así hasta otras tantas dicotomías por establecer; serán los "débiles, indignos, irresponsables, insensatos y malos" los que salgan perdiendo, no porque sean en sí mismos así (que alguno puede que lo sea), sino porque las condiciones imperantes los hacen ser más vulnerables ante las dificultades que el propio sistema educativo y social les imponen por no ser capaces de ajustarse a dichas condiciones. De hecho, resultan ser unas representaciones negativas y arbitrarias de esos sujetos. Entonces llega el momento de vigilarlos y de controlar sus comportamientos recurriendo a las amenazas, a las etiquetas, a la culpabilización o a las expulsiones. Pasan así a ese estado de indeterminación mencionado que se cobija en los espacios intersticiales de los lugares comunes y habitados (clase, espacio de recreo, pasillos...), esos espacios que dan cuenta de la relación permitida entre el lugar y el alumnado, y en los que están aquellos a los que les persigue el estigma del fracaso.

Cresswell (1996) señala que alguien pertenece a un lugar y no a otro, y el lugar de cada persona está vinculado con la relación de esta con las demás, cuyos comportamientos le lleva a ocupar una posición en la estructura social de esos lugares comunes. El alumnado que lleva a cuestas el fracaso escolar está "fuera de sitio" (out of place) aunque esté dentro de esos lugares, es decir, está, al mismo tiempo, dentro y fuera (sin estar físicamente fuera, aunque puede llegar a estarlo 
en los casos de las expulsiones), porque estando dentro "no es mirado" (aunque sea "visto"), "no es escuchado" (aunque sea "oído"). Y llegan a estar "fuera de sitio" porque sigue predominando, por una parte, la tecnificación de las estrategias establecidas como soluciones a la situación del que caerá en el fracaso, y, por otra, una despolitización de las medidas adoptadas para hacer frente al mismo, en tanto en cuanto el "problema" se centraliza en el alumnado y, consecuentemente, el problema pasa a ser individual exigiéndole a este que cambie para poder permanecer dentro del sistema. La responsabilidad también se desplaza, dejando de ser colectiva para ser individual: es responsabilidad del que fracasa enmendar su trayectoria desviada para volver al redil institucionalizado. Sentirse no reconocido, no escuchado, no valorado, sentirse desamparado y marginado es lo ganado por el propio sujeto y, por tanto, es el culpable de su situación de exclusión. Como señala Young (2011), dicha situación se reduce a una cuestión de responsabilidad personal. Queda de este modo desvalido, porque el que se muestra un "anti-sistema", en realidad es un alumnado "sin-sistema", porque el sistema educativo establecido está evidenciando que no tiene capacidad para dar respuesta a sus necesidades, dificultades y circunstancias. En virtud de ello, es un sistema que los margina, que incrementa las desigualdades e impide el logro de la equidad, el bienestar educativo y la justicia. ${ }^{12}$

De este modo, cuando se habla en términos de un alumnado "sin-sistema" y se dirige la mirada de fuera a dentro es posible llegar a entender las acciones marginales, las transgresiones y el asalto al orden establecido que realiza aquel, aunque puedan llegar a ser conductas no aceptables (por ejemplo, pegar o agredir). Se descubre la violencia estructural y simbólica que se ejerce sobre el alumnado que desemboca en una imagen paupérrima, no ya de lo que es (si se llega a reconocer quién es), sino de lo que será, conseguida a partir del desahucio iniciado desde las primeras acciones disruptivas (como gusta llamar ahora a las conductas que rompen la norma establecida) o desde las muestras de rechazo a lo escolar o a lo curricular porque no le da respuesta a sus inquietudes y problemáticas (sociales y familiares); vislumbra el proceso que le ha llevado a interiorizar una imagen devaluada de sí mismo gracias a la desvalorización motivada por las valoraciones externas recibidas (por el profesorado, los iguales, los familiares...); permite ser sensible al desencuentro que existe entre el alumnado y la institución educativa y el sistema escolar que impide que los procesos de negociación sean posibles desde un diálogo con sentido común y con racionabilidad, e igualmente, esta mirada ayuda a cuestionar la absolutidad de lo normalizado y normativizado como lo correcto. En este giro de mirada, juegan un papel relevante las aportaciones analíticas y críticas, como puede ser la teoría de la resistencia y la pedagogía crítica, ya que ayudan a bajar de las macroestructuras, del determinismo económico que parece no admitir el reconocimiento de prácticas alternativas, y de la visión de la escuela como mecanismo exclusivo de reproducción (Apple,1996; Gil Ribero, 2002; Giroux, 1985,1992; Haenfler, 2004; Jerez Mir, 1996;

12 Como apuntan Fallis y Opotow (2003), no es el alumnado el que falla al sistema escolar, sino al revés, son este y las escuelas los que le fallan a este. 
Paris y Ault, 2004; Torres Santomé, 1994; Vasudevan y Campano, 2009; Willis, 1988), al terreno de las realidades concretas en el que el alumnado, con sus experiencias disidentes, genera interrupciones en el funcionamiento establecido como una muestra de oposición individual y propia. Los jóvenes que sufren el fracaso y el abandono escolar se construyen desde posiciones subalternas (Spivak, 1988) que, en su intento de llenar el vacío de su no-representación, de su invisibilización, se van auto-representado en sus propios espacios, que son los espacios intersticiales de los lugares comunes y habitados; espacios que resultan problemáticos y en los que se ponen de manifiesto las contradicciones y los desajustes entre los sujetos y los lugares comunes que responden a un habitus homogenizante. Desde esta mirada encontramos elementos contundentes para poder comprender la concurrencia de una toda una serie de actuaciones, representaciones, actitudes, palabras, silencios y gestos que se agolpan en unos cuerpos carentes de privilegios que intentan, a su vez, hacer su propio camino nadando a contracorriente.

\section{EL CAMPO EPISTÉMICO DEL FRACASO Y ABANDONO ESCOLAR: LA EVIDENCIA DE LO COMPLEJO}

No cabe duda de que el fenómeno del fracaso y del abandono escolar resulta ser en estos momentos actuales uno de los temas de mayor preocupación, especialmente cuando atendemos a las cifras que nos ofrecen los diferentes informes nacionales e internacionales y estudios comparativos. El informe Ivie (Serrano, Soler y Hernández, 2013) señala que las tasas de abandono se sitúan entre las más altas de la Unión Europea (UE). Según Eurostat (2017), nuestro país es uno de los de la Unión Europea con mayor nivel de fracaso escolar. El abandono temprano (quienes no han terminado los estudios de educación secundaria) representa el $20 \%$ de los jóvenes con edades comprendidas entre los 18 y 24 años, y son más los hombres los que abandonan el sistema educativo que las mujeres, $21,8 \%$ y $14,5 \%$, respectivamente. En el reciente informe, Panorama de la Educación. Indicadores de la OCDE 2017 (INEE, 2017) señala que España se sitúa en los primeros puestos en relación a la proporción de adolescentes que no siguen estudiando tras la ESO (Figura 1). Considerando el intervalo de 15-19 años, el porcentaje referido a la tasa de escolarización en España (87\%) es superior a la media de los países de la Organización para la Cooperación y el Desarrollo Económico (OCDE) (85\%), y con respecto a la media de los países de la UE22 (88\%), la diferencia es solo de un punto. Atendiendo a la situación en la que se encuentra el alumnado, el $28 \%$ de los estudiantes españoles no están realizando la educación secundaria, es decir, estamos hablando de abandono.

Por su parte, en el informe Datos y cifras. Curso escolar 2017-2018 (2017b) se recoge que el abandono temprano en España es superior (19\%) a la media de la UE28 (10,7\%) (Figura 2), un porcentaje que se ve algo reducido en el segundo trimestre del presente año (18,2\%), según la Encuesta de Población Activa (EPA).

Aunque estas tasas corresponden a la población comprendida entre los $18 \mathrm{y}$ 24 años y entre los 15 y 19, hemos de señalar que en muchos casos en estas edades se manifiesta lo que se ha venido gestando a lo largo de un proceso que puede ha- 
berse iniciado en la educación primaria. Cuando se presta atención a la construcción de las trayectorias escolares de adolescentes, se pone de manifiesto la existencia de incidentes críticos que han accidentado su proceso educativo, dejando una huella significativa que cobra mayor relieve en la educación secundaria. De este modo,

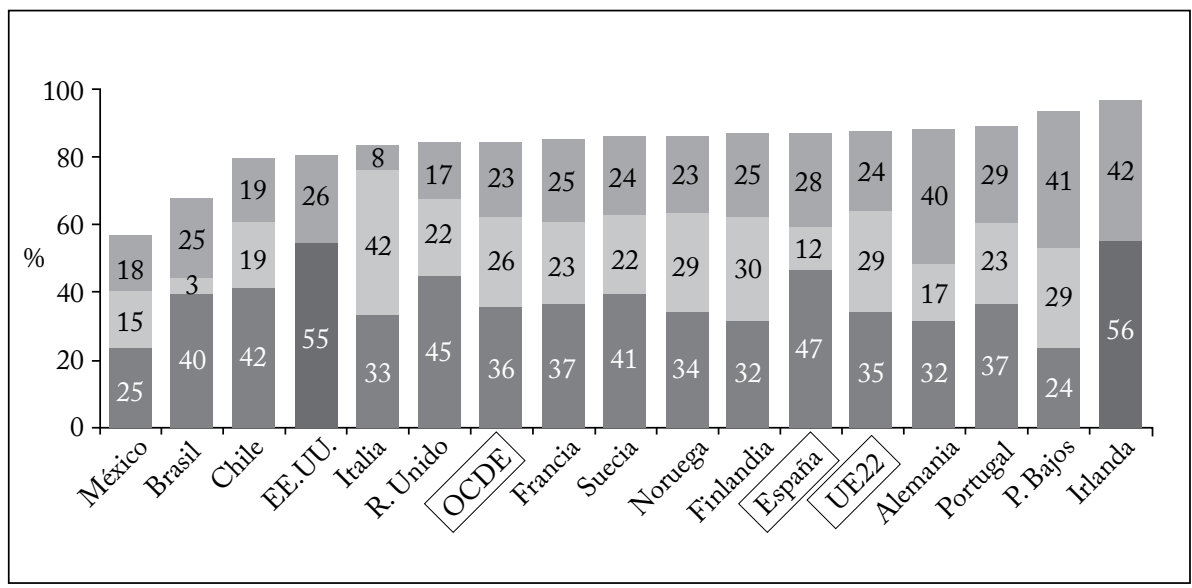

Figura 1 - Tasa de escolarización de la población entre 15-19 años, por nivel y orientación del programa (2015). Fuente: INEE (2017, p. 17). ${ }^{13}$

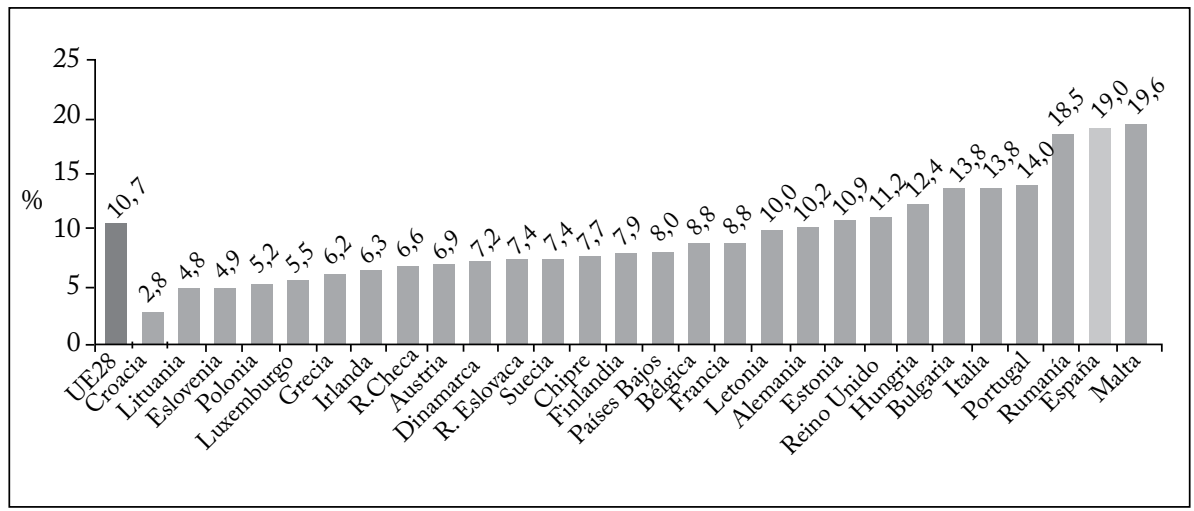

Figura 2 - Abandono educativo temprano. Países de la Unión Europea (2016).

Fuente: Ministerio de Educación, Cultura y Deporte (2017b, p. 25).

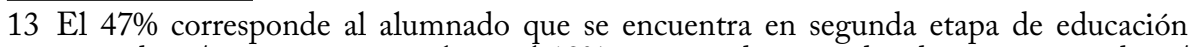
secundaria/programas generales, y el $12 \%$ en segunda etapa de educación secundaria/ formación profesional (INEE, 2017, p. 17). 
el desenganche (Fernández Enguita, 2011; Mena Martínez, Fernández Enguita y Riviére Gómez, 2010) tiene sus desencadenantes en la etapa educativa de primaria. Centrándonos en el contexto de Andalucía, comunidad en la que se ha desarrollado la investigación, cabe decir que es una de las comunidades con mayor índice de abandono (23,5\%). Entre el 2016 y 2017 se ha visto incrementado en 0,4 puntos (de 23,1 se ha pasado a 23,5). Asimismo, el porcentaje de abandono ha sido siempre, en términos generales, inferior entre las mujeres que entre los hombres. Así, en el año 2016, el 15,1\% corresponde a las mujeres y el 22,7\% a los hombres; una diferencia que se mantiene prácticamente en casi todas las comunidades autónomas (Ministerio de Educación, Cultura y Deporte, 2017a).

Ahora bien, al margen de este hecho, y de la utilidad de tales informes, no podemos dejar en el olvido que actualmente se precisa de cifras apoyadas en resortes estadísticos para dar credibilidad y rigor a una situación entendida como problemática, y también para poder realizar comparaciones entre realidades diferentes y desiguales. Estos porcentajes son el instrumento preferido con el que algunos medios de comunicación y las élites dominantes se empeñan en vendernos la situación poco favorable en la que parece encontrarse España, cuando la distancia con la media de la OCDE y la UE no es tan llamativa; la diferencia de cuatro o cinco puntos sobre el total no es para que salten las alarmas, pero así sucede. Igualmente podemos considerar, por ejemplo, que la diferencia con respecto a Noruega (23\%) es de solo cinco puntos (28\%), cuando el sistema educativo español tiene un gasto por alumno que es casi la mitad que el de Noruega. Si tomamos Alemania, el porcentaje de jóvenes que no cursan educación secundaria es del 40\% (recordemos que en España es del 28\%), el gasto público total destinado a la educación secundaria en nuestro país es del 3,5\%, inferior al de Alemania, por tanto, con menos dinero se logra una tasa de estudiantes en educación secundaria mayor. Con este juego de cifras y porcentajes, el sistema educativo español llega a ser más víctima que culpable de su actual situación como efecto de la cuantofrenia inculcada por organismos económicos (poderosos) como la OCDE, que hacen que el sistema sea mostrado a la sociedad, tras pasar por un proceso de simplificación que desemboca en un puñado de cifras orquestadas según convenga, como un asunto que requiere de una urgente reforma; reforma, claro está, para seguir fortaleciendo una educación al servicio del poder económico. A su vez, el profesorado también se ve afectado por lo mismo, e igualmente el alumnado, y de modo especial aquel que "no encaja" en el sistema. En este punto, y paradójicamente, ya se olvida que el sistema es víctima y culpable, porque el culpable (pero no víctima porque no se reconoce así) de la situación de "no encaje" que le lleva a desenganche, al fracaso y/o abandono es el alumnado. Diane Ravitch (2013) señala, en relación a las pruebas de evaluación, que los estudiantes que piensan de otra forma a la reconocida como válida y correcta consiguen puntuaciones más bajas, porque se sigue premiando la conformidad y el cumplimiento para obtener las respuestas correctas. El paraíso de las cifras es una versión de la tecnificación de las situaciones educativas y sociales que requiere de medidas técnicas y científicas para su afrontamiento.

Paralelamente a los informes, el terreno de la investigación y el estudio está bastante abonado por trabajos que han puesto su foco de atención en dimensiones 
y factores diferentes. Así, el estudio de Ferrer Esteban, Castel Baldellou y Ferrer Julià (2006) revisa la persistencia de variables fundamentalmente relacionadas con los centros y sus características. Sin embargo, en este estudio se insiste en el nivel socioeconómico y cultural (estatus ocupacional y nivel académico de los padres y de las madres, y las propiedades en casa). Añaden, además, otras variables de no menor importancia como la autoconfianza y las aspiraciones del alumnado. Calero, Choi y Waisgrais (2010) utilizan variables personales, familiares y escolares para los datos recogidos en Programa Internacional para la Evaluación de Estudiantes o Informe 2006 (PISA) llegando a unas conclusiones tajantes como que tales variables determinan de manera taxativa un alto grado el riesgo de fracaso escolar. Otros factores que se aportan tienen que ver con la inmigración (Calero et al., 2007), con el origen social (Bernardi y Requena, 2010), Breen y Jonsson (2005) señalan que el rendimiento académico está segmentado por clases sociales, Casquero y Navarro (2010) analizan el abandono atendiendo a la variable género, y el trabajo de Mora (2010) se centra en los determinantes del abandono escolar. Caben destacar otras investigaciones como las de Entorf y Minoiu (2005), Escudero Muñoz (2005), Escudero Muñoz, González González y Martínez Domínguez (2009), Estrada de Madariaga (2017), Lizasoain et al. (2007), Marchesi (2000, 2003), Moriña Díez (2007) y Rumberger y Ah Lin (2008).

Informes e investigaciones no dejan de ofrecer, digámoslo así, una realidad que se ve obligada a debatirse entre quienes tienen éxito y quienes no (esto es, los que fracasan y pueden llegar a abandonar), entre los incluidos en el sistema y los excluidos, entre si aumenta o disminuye el fracaso y/o el abandono escolar entre unos países y otros, entre unas comunidades y otras, y un largo etcétera que ilustran las diferentes y diversas dicotomías; unas dicotomías, reales, que, sin embargo, más que ayudar a la comprensión fehaciente de lo que ocurre en relación a ese fenómeno, lo que hace es ensombrecer y simplificar el universo determinante del mismo. No podemos negar que es un hecho, una realidad la del fracaso escolar y el abandono que se produce. Algo está pasando. Pero el esclarecimiento de lo que está ocurriendo exige andar por otros terrenos que complementen el corpus científico existente, el cual aporta una fotografía macroscópica del fracaso y del abandono encapsulada en un conglomerado de factores que coloca a los "afectados" en un plano secundario carente, al mismo tiempo, de la trama compleja que configuran los sistemas educativo y político, y los contextos socioculturales y familiares. Andar por otros terrenos nos puede ayudar a descubrir nuevos hechos y nuevos elementos focalizando la mirada en aspectos que ya han sido mirados pero han pasado desapercibidos o fueron considerados irrelevantes.

\section{LA EVIDENCIA DE LO COMPLEJO: LA INTERSECCIONALIDAD}

De una u otra manera, podemos decir que los estudios disponibles configuran una trama compleja de variables que contemplan las características del sistema educativo, de las familias, de los centros educativos, de las aulas y del propio alumnado. Este hecho ya nos avisa de que el fracaso no es una cuestión inherente al propio estudiante. Esta constancia exige incorporar un marco interpretativo que ayude a 
explicar y a comprender tal hecho. Dicho marco es la interseccionalidad (Hill Collins, 1990, 2009) que nos sitúa en los diferentes factores y sistemas que determinan el fracaso y el abandono escolar desde la consideración de la interdependencia, la interacción y la intersección entre los mismos, que lo hace, además, desde la óptica de los dispositivos que contribuyen al disfrute de privilegios o a todo lo contrario, a experimentar desigualdad, discriminación y exclusión. El alumnado perteneciente a la categoría de "fracaso escolar" precisamente se sitúa en esa intersección, y es por ello por lo que nuestra mirada se sitúa en el alumnado para poder comprender la manera en la que factores y sistemas inciden e interconectan con sus experiencias (familiares, escolares, sociales, etc.); experiencias insertas en contextos sociales (y educativos) desiguales caracterizados por la complejidad, y en los que los sistemas que generan desigualdad se unen (sistema educativo, sistema económico, sistema social, sistema patriarcal).

No es posible buscar razones explicativas tomando cada factor $\mathrm{y}$ sistema por separado, porque es limitante para la comprensión del fracaso y del abandono, y sobre todo de los propios sujetos; es necesario asumir una perspectiva multifactorial y multidimensional para poder comprender tales fenómenos pero siempre desde esa interseccionalidad que dibuja las formas de dominio bajo las que los jóvenes "sin-sistema" construyen sus identidades (género, cultura, etnia, clase social, etc.). $\mathrm{Y}$ es preciso asumir y posicionarnos en la perspectiva de la interseccionalidad que nos ofrece Hill Collins (1990) para evitar que se naturalicen la desigualdad, la inequidad y la injusticia, y también para poder entender la presencia de la resistencia en las instituciones educativas, como también en otros contextos (familiar y social). De ahí que sea necesario explicar tales fenómenos desde la imbricación de factores e incidentes múltiples que convergen a lo largo de la trayectoria personal y escolar de los sujetos.

Desde esta consideración, la dimensión personal e individual no es determinante para la concurrencia del fracaso y/o abandono, pues no depende directa ni exclusivamente de las circunstancias individuales; más bien son el resultado $\mathrm{y}$ el efecto de la violencia estructural que ejercen los sistemas (social, educativo, político y económico) sobre dichas circunstancias y que obliteran el disfrute de los derechos obligatorios que ha de garantizar el Estado de bienestar para con los sujetos. De tal manera, y de modo inevitable, el fracaso y el abandono se tornan en expresiones de la exclusión que experimentan los sujetos abocados a ese fracaso y/o abandono al que han sido arrojados. Resulta por tanto necesario tomar como foco central de análisis a los sujetos encarnados en sus propias experiencias y vivencias, porque las identidades que van construyendo estarán atravesadas por múltiples factores opresores que impiden el acceso a derechos y oportunidades. La asunción de la interseccionalidad para el análisis del fracaso y del abandono es clave para el esclarecimiento de las diferentes discriminaciones y desventajas que se pueden dar como efecto de la interrelación entre factores y sistemas. Existen diversas formas de discriminación, tales como el patriarcado, el racismo, la xenofobia, la aporofobia, el colonialismo, la opresión de clase, pero también lo es el fracaso escolar (forma de discriminación que interconecta con las anteriores), ya que todas estas 
formas producen desigualdades y exclusión al definir la posición de los sujetos en las estructuras sociales institucionalizadas.

El enfoque de la interseccionalidad trae consigo la matriz de dominación que Hill Collins (1990, p. 299) define como "la organización general de las relaciones jerárquicas de poder en cualquier sociedad”. Señala, a su vez, que "cualquier matriz particular de dominación se organiza a través de cuatro dominios de poder interrelacionados: estructurales, disciplinarios, hegemónicos e interpersonales" (Hill Collins, 1990, p. 276). Estos cuatro dominios son identificables en el análisis del fracaso escolar, y especialmente, de las experiencias del alumnado que se encuentra inmerso en ese proceso. En este sentido, el dominio estructural tiene que ver con las estructuras sociales (como pueden ser la economía, las regulaciones normativas, la política, etc. $)^{14}$ que generan procesos asfixiantes y limitadores que acarrean desigualdad y discriminación. La precariedad laboral, el paro de los progenitores, la escasez de recursos, las condiciones de inhabitabilidad de las viviendas, la pobreza, la marginación política, las medidas de apoyo al alumnado que perpetúa un modelo excluyente, la política educativa, etc., son ejemplos de este dominio estructural que intervienen en la producción de la opresión, y también en el desarrollo de estrategias de resistencia.

No ajeno a este dominio es el disciplinario que tiene como misión controlar, gestionar y organizar el comportamiento recurriendo al habitus, a la rutina, al orden, al control, a la vigilancia. Estos elementos no son extraños a cualquier institución educativa, los cuales se orquestan para que ésta alcance sus propósitos y el alumnado asimile los dispositivos necesarios para actuar según la norma y pueda responder a las exigencias establecidas.

Dichos elementos, a su vez, tienen la capacidad de encubrir las dinámicas que generan discriminación y desigualdad recurriendo al discurso que, bajo el estandarte de la calidad y de los buenos resultados, ha resignificado los valores de la igualdad y la equidad; es decir, nos encontramos con prácticas discursivas aparentemente inclusivas que no lo son realmente. Así, por ejemplo, las expulsiones de los centros del alumnado considerado "fracasado" se toman como una medida que le va a ayudar a recapacitar sobre lo realizado. Sin embargo, la "buena intención" de esta medida tiene un doble efecto: uno, hay docentes que lo toma como norma para no tener al alumnado en clase, y en este sentido, el efecto es excluyente, propio de este dominio, y otro, el alumnado busca ser expulsado, y en tal caso esta estrategia es una muestra de su resistencia. ${ }^{15}$ Por su parte, el dominio interpersonal hace referencia a las relaciones personales y a las interacciones que se dan en la vida cotidiana del alumnado, tanto en las instituciones educativas como en otros escenarios sociales, y que inciden de un modo u otro en la construcción de su sí mismo, es decir, cómo se ve, cómo se conceptualiza, cómo se representa, etc., y en este proceso constitutivo inciden directamente los otros (profesorado,

14 Ravitch (2013, s/p.) señala que "mejorar la calidad de vida de casi un cuarto de los estudiantes que viven en la pobreza mejoraría sus resultados académicos".

15 Por eso la interseccionalidad es una herramienta que permite comprender también los procesos de resistencia que se generan como consecuencia de la matriz de dominación. 
amigos, compañeros, familia, etc.): "los maestros se creen que yo no sirvo para nada", ${ }^{16}$ "soy la oveja descarrilada", ${ }^{17}$ "soy un caso perdido", ${ }^{18}$ "soy muy malo", ${ }^{19}$ etc. Se pone así de manifiesto que esa conceptualización que expresan no se produce aisladamente, sino, y como argumenta Taylor (2009, p. 63), de forma dialógica. "Siempre definimos nuestra identidad en diálogo con las cosas que nuestros otros significantes desean ver en nosotros, y a veces en lucha con ellas", es decir, la identidad que estos jóvenes construyen depende de sus "relaciones dialógicas con los demás" (Taylor, 2009, p. 65). Consecuentemente, estas relaciones pueden llegar a ser el espacio para el ejercicio del poder y de la dominación.

Finalmente, el dominio hegemónico incide en los anteriores y tiene que ver con el lenguaje que empleamos, los libros de textos, la cultura oficial, la organización de los espacios y los tiempos, las relaciones entre profesorado-alumnado, etc. Dominan los códigos elaborados que excluyen a quienes se manejan con códigos restringidos, prevalece la cultura oficial y curricular sobre la cultura vinculada a las experiencias y a la vida cotidiana del alumnado, se antepone una relación menos cerca y amigable y más disciplinar a otra que escucha las circunstancias particulares, se priorizan las voces de las culturas hegemónicas al tiempo que se silencian aquellas cuyas culturas corresponden a grupos sociales marginados o a minorías que no tienen el mismo reconocimiento, se valora más y mejor aquellas personalidades que se van ajustando a las exigencias de un sistema neoliberal y conservador - las personalidades neoliberales y neocolonialistas de las que habla Torres Santomé (2017), que aquellas otras que se muestran rebeldes, disidentes o insurgentes.

\section{NUEVOS CAMINOS Y NUEVAS EXIGENCIAS}

Tomar la interseccionalidad como herramienta de análisis del fenómeno del fracaso y del abandono escolar supone asumir una mirada política que desmitifica la despolitización que se vierte en las decisiones que se toman al respecto, reconociendo de partida que en sí misma resulta compleja en tanto que supone y exige manejarse con múltiples categorías, tanto a nivel inter como intra (McCall, 2005). Esta asunción como compromiso pone de manifiesto que no se trata de un fenómeno neutral, estático, cerrado que se produce al margen de las políticas educativas y de las políticas que dan cuenta de la gestión y la organización de las instituciones educativas. No hay una independencia ideológica sino un aparato ideológico que va configurando un tipo de sujeto, a imagen y semejanza de las necesidades de aquel. Aquellos sujetos que no encajan en ese tipo de sujeto que se va configurando

16 Testimonio de un adolescente de 15 años, repetidor de $1^{\circ}$ ESO (actualmente cursa Programa de Refuerzo, Orientación y Apoyo - PROA).

17 Testimonio de un adolescente de 17 años, repetidor de $2^{\circ}$ y $3^{\circ}$ de ESO (cursa $4^{\circ}$ de ESO).

18 Testimonio de un adolescente de 17 años, repetidor de $1^{\circ}$ y $2^{\circ}$ de ESO (realiza curso privado de profesional).

19 Testimonio de un adolescente de 17 años, repetidor de $3^{\circ}$ y $4^{\circ}$ de ESO (cursa $4^{\circ}$ de ESO). 
terminan siendo la expresión más evidente y contundente de la intersección de las formas de dominio que actúan tanto sobre los que no "encajan" como sobre los que sí se amoldan; la diferencia entre unos y otros es que los primeros se resisten, y en esta resistencia intentan encontrar la vía para que les ayuden a dar respuestas a sus necesidades, sus dificultades, sus intereses, etc.

Porque esta resistencia hemos de entenderla como un reclamo para obtener, de algún modo, el reconocimiento que los jóvenes perciben que no tienen. Son llamadas de atención que sirven para decir "aquí estoy yo y mis circunstancias", pese a que sean unas llamadas que resultan incómodas, inapropiadas o incorrectas según las reglas del orden institucional. Ese reconocimiento está vinculado a su identidad, en la medida en que en esa relación dialógica se pone en juego o no el reconocimiento que los demás hacen de los jóvenes que no "encajan". Puede darse el caso de ausencia de reconocimiento o ser el falso (estereotipado y pr juicioso) reconocimiento por parte de los demás. De este modo, y como señala Taylor (2009, p. 53-54),

un individuo o grupo de personas puede sufrir un verdadero daño, una auténtica deformación si la gente o la sociedad que lo rodean le muestran, como reflejo, un cuadro limitativo, o degradante o despreciable de sí mismo. El falso reconocimiento o la falta de reconocimiento pueden causar daño, pueden ser una forma de opresión que subyugue a alguien en un modo de ser falso, deformado y reducido.

El autodesprecio al que pueden llegar los jóvenes viene como efecto de estas prácticas. Por ello, la resistencia emerge de la matriz de dominación, en la que se configura el fracaso y abandono, como reclamo de un reconocimiento legítimo como sujeto que desea ser agente político (reconocimiento político).

Asimismo, la incorporación de esta herramienta, a su vez, supone poner en valor las voces de los adolescentes, los jóvenes "sin-sistema", que, de un modo u otro, experimentan la exclusión y la desigualdad (Calderón Almendros, 2014, 2016a, 2016b; Fernández Enguita, 2011; Fernández Enguita, Mena Martínez y Riviére Gómez, 2010; Gamoran, 2001; Jiménez, Luengo y Taberner, 2009; Mena Martínez, Fernández Enguita y Riviére Gómez, 2010; O’Connor, 2001; Parrilla Latas, Gallego Vega y Moriña Díez, 2010; Romero Sánchez y Hernández Pedreño, 2018; Salvá-Mut, Oliver-Trobat y Comas-Forgas, 2014; Sánchez Alex, 2016; Smyth y Hattam, 2004; Susinos Rada y Parrilla Latas, 2008); son las voces subalternas (Apple y Buras, 2006; Spivak, 1988) silenciadas en el currículum, en los lugares comunes y habitados. Desde el conocimiento de las experiencias del alumnado es posible conocer el proceso complejo que le ha llevado a desengancharse paulatinamente - dropping out (Smyth y Hattam, 2004) - , a romper los vínculos estructurales y relacionales, a volverle la cara al sistema educativo, a mostrar su resistencia y desaprobación a ese sistema que no le otorga valor, reconocimiento ni garantías de equidad porque es portador de unos códigos y de una cultura que no se corresponden con el canon escolar autorizado, a construir una imagen de sí mismo negativa o con pocas esperanzas de lograr algo en el futuro. Esto implica reconocer 
a los jóvenes "sin-sistema" como sujetos capaces de dar cuenta de su propia situación y de su vivencia del desenganche/fracaso/abandono, capaces de aportar su propia visión e interpretación de las mismas, y capaces de poner de manifiesto cómo su subjetividad, su sí mismo que construye, se ve atrapada por la matriz de dominación. En este sentido, ponemos en alza el valor del conocimiento situado (Haraway, 1995) que los propios sujetos construyen desde su realidad situada que pertenece a unos contextos determinados por factores políticos, ideológicos, culturales, sociales, económicos; una realidad situada que no deja de ser un posicionamiento político. Desde esta mirada, podemos entender la resistencia como una oposición personal e individual a la dominación, que en palabras de Taylor y Whittier (1992, p. 117) sería la "politización del yo".

\section{REFERENCIAS}

Apple, M. W. Politica cultural y educación. Madrid: Morata, 1996.

Apple, M.W.; Buras, K. (Eds.). The subaltern speak: curriculum, power and educational struggles. London: Routledge, 2006.

Bernardi, F.; Requena, M. Inequality in educational transitions: the case of postcompulsory education in Spain. Revista de Educación, Madrid: MECD, p. 93-118, 2010. (Número extraordinario 2010: Abandono temprano de la educación y la formación: cifras y políticas).

Breen, R.; Jonsson, J. O. Inequality of opportunity in comparative perspective: Recent research on educational attainment and social mobility. Annual Review of Sociology, Palo Alto, CA: [s.n.], v. 31, p. 223-243, Aug. 2005. https://doi.org/10.1146/annurev. soc.31.041304.122232

Calderón Almendros, I. Sin suerte, pero guerrero hasta la muerte: pobreza y fracaso escolar en una historia de vida. Revista de Educación, Madrid: MECD, n. 363, p. 184209, enero/abr. 2014. https://doi.org/10.4438/1988-592X-RE-2012-363-177 UOC, 2016a.

. Fracaso escolar y desventaja sociocultural. Una aproximación biográfica. Barcelona:

. Liberarse de la escuela. Historia de vida de Elena. Málaga: RIUMA, 2016b.

Calero, J. et al. Desigualdades socioeconómicas en el sistema educativo español. Madrid: Ministerio de Educación y Ciencia; Centro de Investigación y Documentación Educativa, 2007.

Calero, J.; Choi, A.; Waisgrais, S. Determinantes del riesgo de fracaso escolar en España: una aproximación a través de un análisis logístico multinivel aplicado a PISA 2006. Revista de Educación, Madrid: MECD, p. 225-256, 2010. (Número extraordinario 2010: Abandono temprano de la educación y la formación: cifras y políticas).

Casquero, A.; Navarro, M. Determinantes del abandono escolar temprano en España, un análisis por género. Revista de Educación, Madrid: MECD, p. 191-213, 2010. (Número extraordinario 2010: Abandono temprano de la educación y la formación: cifras y políticas). 
CREsswell, T. In placelout of place: geography ideology and transgression. Minneapolis: University of Minnesota Press, 1996.

Entorf, H.; Minoiu, N. What a difference Immigration Policy Makes: a comparison of PISA scores in Europe and traditional countries of Immigration. German Economic Review, [S.1.]: John Wiley \& Son, v. 6, n. 3, p. 355-376, Aug. 2005. https://doi. org/10.1111/j.1468-0475.2005.00137.x

Escudero Muñoz, J. M. Fracaso escolar, exclusión educativa: ¿De qué se excluye y cómo? Profesorado: Revista de Curriculum y Formación del Profesorado, Granada: Universidad de Granada, v. 9, n. 1, p. 1-23, jul. 2005.

Escudero Muñoz, J. M.; González González, M. T.; Martínez Domínguez, B. El fracaso escolar como exclusión educativa: comprensión, políticas y prácticas. Revista Iberoamericana de Educación, [S.1.]: OEI; CAEU, v. 50, p. 41-64, mayo/agosto 2009.

Estrada de Madariaga, M. Estudio descriptivo sobre el abandono escolar temprano e influjo de variables personales y socioculturales en la Ciudad Autónoma de Melilla. Tesis (Doctoral) - Universidad de Granada, Granada, 2017.

Eurostat. Early leavers from education and training, 2017. Eurostat: Oficina Europea de Estadística, 2017. Disponible en: <https://bit.ly/1RYpOXy>. Acceso en: 1 jul. 2018. Fallis, R. K.; Оротоw, S. Are students failing school or are schools failing students? Class cutting in high school. Social Issues, Washington, DC: APA, v. 59, n. 1, p. 103-119, enero 2003. 10.1111/1540-4560.00007

Fernández Enguita, M. Del desapego al desenganche y de este al fracaso escolar. Propuesta Educativa, Buenos Aires: FLACSO, v. 1, n. 35, año 20, p. 85-94, jun. 2011.

Fernández Enguita, M.; Mena Martínez, L.; Riviere Gómez, J. Fracaso y abandono escolar en España. Barcelona. Fundación "La Caixa", 2010. (Colección Estudios Sociales, n. 29).

Ferrer Esteban, G.; Castel Baldellou, J. L.; Ferrer Julià, F. Las desigualdades del sistema educativo a través del estudio PISA 2003. Revista de Educación, Madrid: MECD, p. 399-428, 2006. (Número extraordinario 2006: PISA - Programa para la Evaluación Internacional de Alumnos).

Finn,J.D.; Rock, D. A. Academic success among students at risk for school failure. Journal of Applied Psychology, Washington, DC: APA, v. 82, n.2, p. 221-234, abr. 1997. Disponible en: <https://www.ncbi.nlm.nih.gov/pubmed/9109280>. Acceso en: 29 sept. 2017.

Gamoran, A. American schooling and educational inequality: a forecast for the 21st century. Sociology of Education, Washington, DC: ASA, v. 74, p. 135-153, 2001. (Extra Issue: Current of Thought: Sociology of Education at the Dawn of the 21st Century). $10.2307 / 2673258$

Gil Ribero, J. La educación como espacio de resistencia y transformación social. Filosofía, politica y economía en el Laberinto, Málaga: Universidad de Málaga, n. 10, p. 69-77, oct. 2002. Disponible en: <https://bit.ly/2LFRciL>. Acceso en: 25 oct. 2017.

Gimeno Sacristán, J. El alumno como invención. Madrid: Morata, 2003.

Giroux, H. Teoría y resistencia en educación. Una pedagogía para la oposición. México: Siglo XXI Editores, 1992. 
Teorías de la reproducción y la resistencia en la nueva sociología de la educación: un análisis crítico. Cuadernos Políticos, [S.1.]: Era Editorial, n. 44, p. 36-65, jul./dic. 1985 (Publicado en Harvard Educational Review, v. 53, n. 39, p. 257-29, 1983).

Haenfler, R. Rethinking subcultural resistence. Core Values of the Straight Edge Movement. Journal of Contemporary Ethnography, [S.L.]: Sage, v. 33, n. 4, p. 406-436, ago. 2004. 10.1177/0891241603259809

Haraway, D. Ciencia, cyborgs y mujeres. La reinvención de la naturaleza. Madrid: Cátedra, 1995.

Hill Collins, P. Another kind of public education. Race, Schools, the media and democratic possibilities. Boston: Beacon, 2009.

Black Feminist Thought. Knowledge, consciousness, and the politics of empowerment. London: Routledge, 1990.

INEE - Instituto Nacional de Evaluación Educativa. Panorama de la Educación. Indicadores de la OCDE 2017. Informe Español. Madrid: Ministerio de Educación, Cultura y Deporte; Secretaría de Estado de Educación; Formación Profesional y Universidades Dirección General de Evaluación y Cooperación Territorial, 2017.

Jerez Mir, R. El sistema escolar ante los retos del mundo actual: una aproximación sociológica. Revista de Educación, Madrid: MECD, n. 310, p. 241-259, mayo/ agosto 1996.

Jiménez, M.; Luengo, J.; TABerner, J. Exclusión social y exclusión educativa como fracasos. Conceptos y líneas para su comprensión en investigación. Profesorado - Revista de Currículum y Formación del Profesorado, Granada: Universidad de Granada, v. 13, n. 3, p. 11-49, 2009. Disponible en: <http://www.ugr.es/ recfpro/rev133ART1.pdf>. Acceso en: 29 sept. 2017.

LeE, C. D. Historical Evolution of Risk and Equity: Interdisciplinary Issues and Critiques. Review of Research in Education, Washington, DC: Sage, n. 33, p. 63-100, Mar. 2009. 10.3102/0091732X08328244.

Lizasoain, L.; Joaristi, L.; Lukas, J. F.; Santiago, K. Efectos contextuales del nivel socioeconómico sobre el rendimiento académico en la educación secundaria obligatoria en la Comunidad Autónoma Vasca (España). Estudio diferencial del nivel socioeconómico familiar y el del centro escolar. Education Policy Analysis Archives/ Archivos Analiticos de Politicas Educativas, Tempe, AZ: Arizona State University, v. 15, p. 1-37, enero 2007.

Marchesi, Á. El fracaso escolar en España. Madrid: Fundación Alternativas, 2003. Documento de Trabajo 1/2003. Disponible en: <https://bit.ly/2IwasKk>. Acceso en: 29 sept. 2017.

. Un sistema de indicadores de desigualdad educativa. Revista Iberoamericana de Educación, [S.1.]: OEI; CAEU, n. 23, p. 135-163, mayo/agosto 2000.

Martínez Reguera, E. Con los niños no se juega. Madrid: Editorial Popular, 2007.

Mccall, L. The complexity of intersectionality. Signs: Journal of Women in Culture and Society, Chicago: University of Chicago, v. 30, n. 3, p. 1.771-1.800, 2005. https://doi. org/10.1086/426800 
Mena Martínez,L.; Fernández Enguita, M.; Riviére Gómez,J.Desenganchados de la educación: procesos, experiencias, motivaciones y estrategias del abandono y del fracaso escolar. Revista de Educación, Madrid: MECD, p. 119-145, 2010. (Número extraordinario 2010: Abandono temprano de la educación y la formación: cifras y políticas).

Ministerio de Educación, Cultura y Deporte. Sistema Estatal de Indicadores de la Educación 2017. Madrid: Ministerio de Educación, Cultura y Deporte; INEE; Subdirección General de Estadística y Estudios, 2017a. Disponible en: <https://bit. ly/2GLAWdJ>. Acceso en: 1 jul. 2018.

. Datos y cifras. Curso escolar 2017-2018. Madrid: Ministerio de Educación, Cultura y Deporte; Secretaría General Técnica; Subdirección General de Documentación y Publicaciones, 2017b. Disponible en: <https://bit.ly/2k82tfH >. Acceso en: 1 jul. 2018. Mora, A.J. Determinantes del abandono escolar en Cataluña, más allá del nivel socioeconómico de las familias. Revista de Educación, Madrid: MECD, n. 1, p. 171-190, 2010. (Número extraordinario 2010: Abandono temprano de la educación y la formación: cifras y políticas).

Moriña DíEz, A. M. La exclusión social: análisis y propuestas para su prevención. Madrid: Fundación Alternativas, 2007.

O'ConNor, C. Making sense of the complexity of social identity in relation to achievemt: a sociological challenge in the new millennium. Sociology of Education, Washington, DC: ASA, n. 74, p. 159-168, 2001. (Extra Issue: Current of Thought: Sociology of Education at the Dawn of the 21st Century). 10.2307/2673260

Paris, J. M; Ault, M. Subcultures and political resistance. Peace Review, United States: Taylor \& Francis, v. 16, n. 4, p. 403-407, dic. 2004. Disponible en: <https://bit. ly/2LSPIOP>. Acceso en: 10 oct. 2017. https://doi.org/10.1080/1040265042000318617 Parrilla latas, Á.; Gallego Vega, C.; Moriña Díez, A. El complicado tránsito a la vida activa de jóvenes en riesgo de exclusión: una perspectiva biográfica. Revista de Educación, Madrid: MECD, n. 351, p. 211-233, enero/abr. 2010.

Perrenoud, Ph. La construcción del éxito y del fracaso escolar. Madrid: Morata, 1996.

Ravitch, D. Lo que necesitas saber sobre los resultados del informe PISA. Huffost, Edition ES, 2013. Disponible en: <https://bit.ly/2JSXuqk>. Acceso en: 15 nov. 2017.

Romero Sánchez, B. E.; Hernández Pedreño, M. Análisis de las causas endógenas y exógenas del abandono escolar temprano: una investigación cualitativa. Educación XX1, Madrid: UNED, 2018 (en prensa). https://doi.org/10.5944/educxx1.21351

Rumberger, R.; Ah Lin, S. Why students drop out of school: A review of 25 years of research. California Dropout Research Project Report \#15Santa Barbara: University of California, oct. 2008. Disponible en: <https://bit.ly/2A9xR4O>. Acceso en: 10 oct. 2017.

Salvá-Mut, FR.; Oliver-Trobat, M. F.; Comas-Forgas, R. Abandono escolar y desvinculación de la escuela: perspectiva del alumnado. Magis: Revista Internacional de Investigación en Educación, Bogotá: Pontificia Universidad Javeriana, v. 6, n. 13, p. 129142, 2014. Disponible en: <https://bit.ly/2mLgvSt>. Acceso en: 15 jul. 2018. 
Sánchez Alex, S. Análisis del fracaso escolar a través de las historias de vida de personas adultas en educación secundaria. Tesis (Doctoral) — Universidad de Cádiz, Cádiz, 2016.

Serrano, L.; Soler, A.; Hernández, L. El abandono educativo temprano: análisis del caso español. Barcelona: Instituto Valenciano de Investigaciones Económicas, 2013.

Sмyтн, J.; Наттам, R. Dropping out, drifting off, being excluded. Becoming somebody without school. New York: Peter Lang, 2004.

Spivak, G. CH. ¿Puede hablar el sujeto subalterno? Orbis Tertius, Buenos Aires, UNLP, v. 3, n. 6, p. 1-44. 1998. Disponible en: <https://bit.ly/2LoKez7>. Acceso en: 29 sep. 2017.

Susinos Rada, T; Parrilla Latas, Á. Dar la voz en la investigación inclusiva. Debates sobre inclusión y exclusión desde un enfoque biográfico-narrativo. REICE - Revista Electrónica Iberoamericana sobre Calidad y Eficacia Escolar, Madrid: REICE, v. 6, n. 2, p. 157-171, 2008. Disponible en: <https://bit.ly/2LAOlaF>. Acceso en: 27 nov. 2017.

TARAbini, A. La meritocracia en la mente del profesorado: un análisis de los discursos docentes en relación al éxito, fracaso y abandono escolar. Revista de Sociología de la Educación (RASE), València: Universitat de València, v. 8, n. 3, p. 349-360. 2015. Disponible en: <https:/ojs.uv.es/index.php/RASE/article/view/8389>. Acceso en: 20 jul. 2018.

TAYLOR, Ch. El multiculturalismo y "la politica del reconocimiento". México: FCE, 2009. (Colección Popular).

Taylor, V.; Whittier, N. E. Collective identity in social movement communities: Lesbian feminist mobilization. In: Morris, A. D.; Mueller, C. M. (Eds.). Frontiers in social movement theory. New Haven, CT: Yale University Press, 1992. p. 104-129. Torres Santomé, J. El curriculum oculto. Madrid: Morata, 1994.

. Políticas educativas y construcción de personalidades neoliberales y neocolonialistas. Madrid: Morata, 2017.

Valente, J.Á. Poesía completa. Edición de Andrés Sánchez Robayna. Barcelona: Galaxia Gutemberg, 2016.

Vasudevan, L.; Campano, G. The social production of adolescent risk and the promise of adolescent literacies. Review of Research in Education, Washington, DC: Sage, v. 33, n. 1, p. 310-353, Mar. 2009. http://dx.doi.org/10.3102/0091732X08330003

VÁzQuEz-Recio, R. Investigación, género y ética: una triada necesaria para el cambio. Forum Qualitative Sozialforschung/Forum: Qualitative Social Research, Berlin: Freie Universität Berlin, v. 157, n. 2, art. 10, 2014. Disponible en: <https://bit.ly/2v4Yr9v>. Acceso en: 15 jul. 2018.

Willis, P. Aprendiendo a trabajar: cómo los chicos de clase obrera consiguen trabajos de clase obrera. Madrid: Akal, 1988.

Young, I. M. Inclusion and democracy. Oxford: Oxford University Press, 2000. . Responsabilidad por la justicia. Madrid: Morata, 2011. 


\section{SOBRE LAS AUTORAS}

Rosa VÁzQuez-Recio es doctora en filosofía y ciencias de la educación por la Universidad de Cádiz (España). Profesora de la misma institución. E-mail: rmaria.vazquez@uca.es

Mónica María López Gil es doctora en educación por la Universidad de Cádiz (España). Profesora de la misma institución.

E-mail:monica.maria@uca.es

Recebido el 9 de diciembre de 2017 Aprobado el 31 de julio de 2018

No artigo "Interseccionalidad,jóvenes "si sistema" y resistencia.Una mirada diferente del fracaso/abandono escolar”, com número de DOI: 10.1590/S1413-24782018230094, publicado na Revista Brasileira de Educação, v23: e230094, na página 1:

ONDE SE LIA:

Interseccionalidad, jóvenes "si sistema" y resistencia. Una mirada diferente del fracaso/abandono escolar

LEIA-SE:

Interseccionalidad, jóvenes "sin-sistema" y resistencia. Una mirada diferente del fracaso/abandono escolar 